\title{
APLICAÇÃO EM JATO DIRIGIDO EM CANA-DE-AÇÚCAR. II: ESPAÇAMENTO ENTRE BICOS TURBO FLOODJET PARA A APLICAÇÃO DE HERBICIDAS ${ }^{1}$
}

\author{
VALDEMIR A. PERESSIN ${ }^{2}$, TOMOMASS A MATUO ${ }^{3}$, DILERMANDO PERECIN ${ }^{3}$, \\ LUÍS C. PIO ${ }^{4}$ e BENEDITO A. BRAZ ${ }^{5}$
}

RESUMO

\begin{abstract}
Aplicações de herbicidas em estádios mais avançados de desenvolvimento da cana-deaçúcar, em pré ou em pós-emergência inicial das plantas infestantes, podem ser feitas em jato dirigido, para que o produto atinja diretamente o alvo. Com esse fim, ava liaram-se 10 bicos TFVS4 a $30 \mathrm{lbf} / \mathrm{pol}^{2}$, determinando-se as vazões e os padrões de deposição em 6 alturas (entre 9 e $36 \mathrm{~cm}$ ), em mesa de prova com 67 canaletas. Após is so, um programa computacional simulou o padrão de deposição de dois bicos separados entre 20 e 70cm no centro da entre linha da cana-deaçúcar. Os resultados indicaram que as vazões
\end{abstract}

obtidas com bicos individuais apresentaram média de 2,3 1/min, com coeficiente de variação $2,5 \%$ e os padrões de deposição mostraram boa precisão com um intervalo de confiança estreito. Foi possível selecionar as melhores combinações de altura (A) e espaçamento (E) entre dois bicos, para os espaçamentos usuais da cana-de-açúcar; sendo que as melhores combinações mostraram relação E/A próxima de 1,4. Por exemplo, $\mathrm{A}=$ $35 \mathrm{~cm}$ e $\mathrm{E}=50 \mathrm{~cm}$ para cana-de-açúcar a $140 \mathrm{~cm}$ entrelinhas.

Palavras chave: Tecnologia de aplicação, barra de pulverização, Saccharum officinalis.

\begin{abstract}
Post emergence late herbicide application in sugarcane, should be carried out prefereable in inter-row, directing the spray mainly to the weeds. This study was performed aiming to evaluate the use of two FloodJet nozzles at the center of row sugarcane in situation of the most usual sugarcane row spacing in São Paulo State. It was started by the determination of spray pattern of ten FS-VS4 nozzles in a patternator with 67 channels, at six heights. An appropriated computer program simulated the spray deposition from two nozzles separated 20 to $70 \mathrm{~cm}$ in the center of inter-row
\end{abstract}

ABSTRACT

Directed spraying in sugarcane. II. Spacing between FloodJet nozzles for herbicide application. sug arc ane. Results indicated: a) at the started, nozzles output was $2.31 / \mathrm{min}$, with a coefficient of variation of $2.5 \%$, showing good repetitivity and reliability. Regarding to the spray pattern it was observed a go od precision with a narrow confidence interval by channel; b) it was observed that in the best combination of the relation nozzle spacing by height was about 1.4. For example, nozzle spacings at $50 \mathrm{~cm}$ and nozzle hei ght at $35 \mathrm{~cm}$, for sugarcane inter-row at $140 \mathrm{~cm}$.

Key words: Spraying tecnology, spray boom, Saccharum officinalis, nozzle.

1 Recebido para publicação em 09/05/96 e na forma revisada em 19/11/96.

2 Instituto Agronômico, Pesquisador da Divisão de Plantas Alimentícias Básicas, Av. Barão de Itapura, 1481, Caixa Postal, 28, CEP 13020-432, Campinas-SP.

3 FCAV/UNESP, Rodovia Carlos Tonani, Km 5, Jaboticabal-SP.

4 Herbicat Ltda., Catanduva-SP.

5 ISK-Biosciences, Av. Nove de Julho, 5617, CEP 01407-912, São Paulo-SP. 


\section{INTRODUÇÃO}

O controle químico das plantas daninhas em estádios mais avançados de desenvolvi mento da cana-de-açúcar, seja em pré ou pós-emergência das plantas infestantes, pode ser realizado em jato dirigido para que o herbicida seja direcionado predominantemente para o alvo.

Para se obter a máxima eficiência biológica de um herbicida, é necessário o conhecimento de seu mecanismo de ação, bem como das possíveis interações deste produto com o alvo.

Desta forma, é de importância o conhecimento das caracterís ticas do bi co pulverizador, o qual influenciará a vazão, o tamanho das gotas, o ângulo de abertura do jato e o padrão de distribuição. O bico é responsável também por outras duas funções fundamentais na pulverização: controlar a saída do líquido na quantidade desejada por unidade de área e espalhar as gotas formadas dentro de uma área delimitada (Silva, 1982).

Assim, a escolha incorreta dos bicos constitui-se numa das principais causas respon sáve is por fracas sos na aplicação de defensivos (Matuo, 1982).

Os bi cos de pulve rização poss ue m padrões de distribuição característicos, os quais determinam a altura deles em relação ao alvo, bem como seu espaçamento numa barra de pulverização. $\mathrm{O}$ conhecimento da distribuição quantitativa da calda pulverizada ao longo da fai xa de de posição é, portanto, de grande importância no estudo de uma pulverização.

Matuo et al. (1994) estudaram padrões de distribuição de combinações de bicos TwinJet, visando o seu emprego em cana-de-açúcar nas aplicações em pós-emergência e em jato dirigido, com a utilização do herbicida MSMA. É oportuno relatar, que no geral, esse herbicida possui absorção praticamente só foliar e uma transloc ação apossimplasto restrita, sendo normalmente aplicado em pós-emergência média a tardia e em mistura de tanque com diuron, portanto necessita de um bico que possua boa penetração em folhagens densas e gotas menores para proporcionar uma boa cobertura foliar.

Por outro lado, nas aplicações em jato di rigido em pré ou pós in icial das plantas infestantes, onde se utilizam principalmente herbicidas que apresentam absorção foliar e radicul ar (ame trina, di uron, hex azi none, ox ifluorfen, etc.) a utilização do bico Turbo Floodjet pode ser interessante; daí, o objetivo de simular essa utilização, nos espaçamentos usuais para a cultura da cana-de-açúcar.

\section{MATERIAL E MÉTODOS}

Este trabal ho foi de senvolvido no laboratório da Faculdade de Ciências Agrárias e Veterinárias, da Universidade Estadual Paulista Câmpus de Jaboticabal.

O bico TF-SV4 foi selecionado dentre os bi cos hi dráulicos di sponívei s no mercado brasileiro, em função do tamanho de gotas, do padrão de distribuição e do ângulo de abertura do jato.

Foram aval ia dos 10 ex emplares, do estoque comercial da empresa Herbicat Ltda, determinando-se inicialmente a vazão a 30 $\mathrm{lbf} / \mathrm{pol}^{2}$ e os resultados foram expressos pel a vazão média em litros por minuto e respectivo coeficiente de variação.

Em continuidade à avaliação da qualidade, determinaram-se os padrões da deposição (ou distribuição do pulverizado) em uma mesa de prova construída de acordo com as especificações da ORGANIZACIÓN MUNDIAL DE LA SALUD (1976) para testes de bicos. Essa mesa é constituída de uma chapa de metal corrugado formando canaletas distanciadas de 2,5 $\mathrm{cm}$ entre si, num total de 67 canaletas, tendo um tubo coletor graduado corresponden do a cada canaleta. O bico a ser testado foi centrado na canaleta 33 e, avaliado nas alturas de 9, 16, 19, 26, 29 , e $36 \mathrm{~cm}$ do alvo, sendo que o volume coletado nos tubos, para cada bico, foi padronizado numa unidade de tempo (um minuto) e pressão 
Aplicação em jato dirigido em cana-de-açúcar. 11. Espaçamento entre bicos turbo floodjet para aplicação de herbicidas.

(30 lbf/pol2).

Com os 10 exemplares, foi determinada a precisão do padrão de distribuição do bico para cada altura, através do cálculo do volume médio porcentual de positado em cada canaleta e respectivo inte rvalo de $95 \%$ de confiança. Com esses dados foram construídos gráficos, usando o software SGPLUS. A partir dessas vazões obtidas com os bicos individuais, em cada altura e canaleta, um programa computacional especialmente construído simulou as combinações de dois bicos, dispostos com um certo deslocamento entre eles. Com esses novos dados, foram novamente construíd os gráficos, usando o sof tware SGPLUS. Após a análise dos padrões apresentados pela simulação de dois bicos, tornou-se possível escolher as melhores combinações de altura e espaçamento entre eles para uso em jatos dirigidos.

Para a construção de histogramas representativos da deposição de cada conjunto de dois bicos, foram selecionadas as alturas de 9, 16, $19,26,29$, e $36 \mathrm{~cm}$ e os espaçamentos entre bicos variando entre 20 e $70 \mathrm{~cm}$, de $5 \mathrm{em} 5 \mathrm{~cm}$. As escolhas foram realizadas objetivando-se a aplicação em cana-de-açúcar nos espaçamentos usuais de 90 a $150 \mathrm{~cm}$ entre linhas; procurandose aplicar na linha ao redor de $80 \%$ da dosagem da entrelinha.

\section{RESULTADOS E DISCUSSÃO}

\section{Avaliação do bico hidráulico}

As vazões obtidas com os bicos selecionados (TF-VS4) foram em média de 2,3 1/min, com coef iciente de variação $2,5 \%$, o que indica boa repetitividade e confiabilidade dos dados.

Os padrões de distribuição ou de deposição nas 67 canaletas da mesa de prova, bem como os respectivos intervalos de confiança (segmento vertical), para cada uma das alturas, examinadas estão apresentados na Figu ra 1. De modo geral, há uma boa precisão nos padrões de distribuição determinados, para as diferentes alturas avaliadas, com um intervalo de confiança menor que $0,5 \%$ por canaleta. $\mathrm{O}$ efeito da altura do alvo mostrou apenas um maior espalhamento à medida em que aumentou-se a altura, mostrando que o padrão depende basicamente do tipo de bico e da variabilidade aleatória que existe entre eles (Perecin et al., 1994).

\section{Escolha da combinação de altura e espaçamento para a aplicação em jato dirigido}

Para possível aplicação em jato dirigido, estudaram-se as distribuições de combinações de dois bicos com diferentes deslocamento entre eles, para serem colocados no centro da entrelinha da cana-de-açúcar de maneira similar ao apresentado em Matuo et al. (1994) e descrito na metodologia.

Admitindo-se que a linha da cultura tenha $40 \mathrm{~cm}$ e que ela receba pulverização pelos dois lados, ter-se-á para igualdade em volume na linha e na entrelinha, os valores apresentados na Tabela 1.

$O$ ideal seria uma distribuição achatada ou levemente triangular na área da entrelinha com média levemente superior à calculada $(1,79 \%$ para a cana-de-açúcar plantada a 1,40 m) e inclinada nas caudas tendo a média da linha exatamente na posição central da mesma e com valor levemente inferior ao calculado $(0,89 \%$ para a cana-deaçúcar plantada a $1,40 \mathrm{~m}$ ); uma vez que, na linha, existem efeitos complementares de controle, proporcionados pela maior ou menor capacidade competitiva de cada cultura, conforme o observa do por Lunsford (1983). As sim, pode - se concluir que a capacidade competitiva da cultura na região da linha é maior que na entrelinha, sugerindo-se a utilização de maiores dosagens na en trel in ha em relação à dosagens a se rem aplicadas na linha.

Seguindo esta linha de raciocínio, Matuo et al. (1994) obtiveram bons resultados em canade-açúcar, usando uma dosagem na linha ao redor de $80 \%$ daquela usada na entrelinha, e isso foi posteriormente recomendado na prática (Spraying Systems, 1995).

Usando a quase simetria das curvas e o princípio, descrito, pode-se escolher curvas que se aproximam desse ideal, cuja média na posição da linha é um pouco menor que o valor da Tabela 1, 
de modo que a dosagem aplicada na linha seja um pouco menor que a da entrelinha.

Para a altura de $19 \mathrm{~cm}$ a distribuição só mostrou-se razoável para a referida cultura em espaçamentos estreitos, de $90 \mathrm{~cm}$ ou menos. Para a al tura de $26 \mathrm{~cm}$ foram obtidas distribuições satisfatórias para a cana-de-açúcar a 100 e $110 \mathrm{~cm}$ com os bicos distanciados $35 \mathrm{~cm}(\mathrm{E} / \mathrm{A}=1,35) \mathrm{e}$ $40 \mathrm{~cm}(\mathrm{E} / \mathrm{A}=1,54)$, respectivamente.

Para a altura de $29 \mathrm{~cm}$ foi obtida uma distribuição boa para a cana-de-açúcar no espaçamento de $110 \mathrm{~cm}$ e com os dois bicos no centro da entrelinha distanciados um do outro de $40 \mathrm{~cm}$ o que dá uma relação espaçamento/altura $(\mathrm{E} / \mathrm{A})=$
1,38; para out ros es paçamentos, nes ta altura, foram obtidas combinações para a cana-de-açúcar em 100 e $120 \mathrm{~cm}$ com bicos distanciados $35 \mathrm{~cm}$ $(E / A=1,21)$ e $45 \mathrm{~cm}(E / A=1,56)$, respectivamente.

Para espaçamentos maiores, uma distribuição melhor pode ser obtid a com o bico numa . altura maior; por exemplo com $36 \mathrm{~cm}$ e bicos distanciados de $50 \mathrm{~cm}(\mathrm{E} / \mathrm{A}=1,39)$, tem-se uma combinação boa para cana espaçada a $140 \mathrm{~cm}$. Outras combinações razoáveis, nesta altura foram: can a $120 \mathrm{~cm} \mathrm{E}=40 \mathrm{~cm}(\mathrm{E} / \mathrm{A}=1,11)$, can a $130 \mathrm{~cm} \mathrm{E}=45 \mathrm{~cm}(\mathrm{E} / \mathrm{A}=1,25)$, cana a $150 \mathrm{~cm} \mathrm{E}=$ $55 \mathrm{~cm}(\mathrm{E} / \mathrm{A}=1,53)$. As Figuras 2 e 3 ilustram algumas dessas combinações.
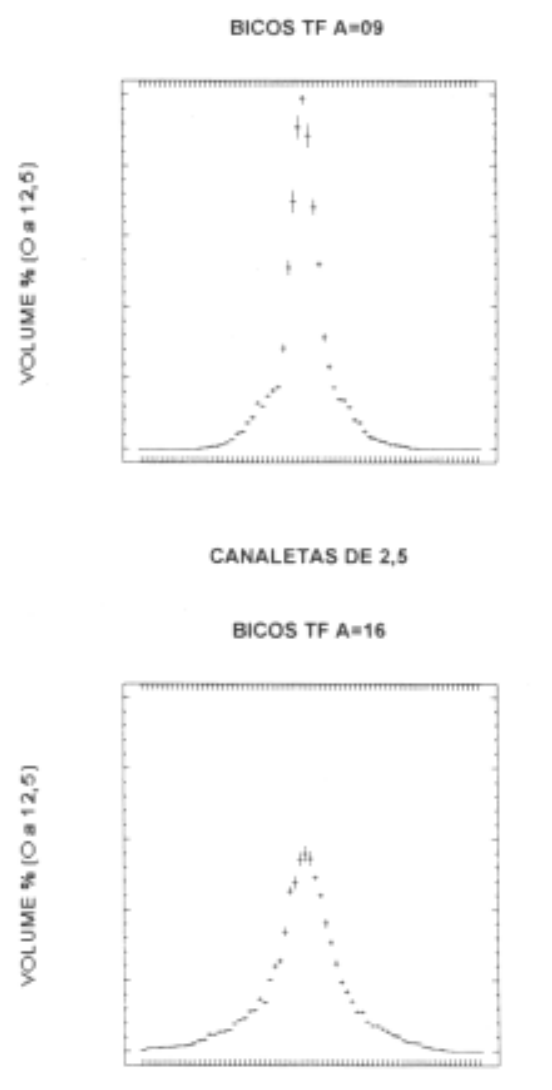

CANALETAS DE $\mathbf{2 , 5}$

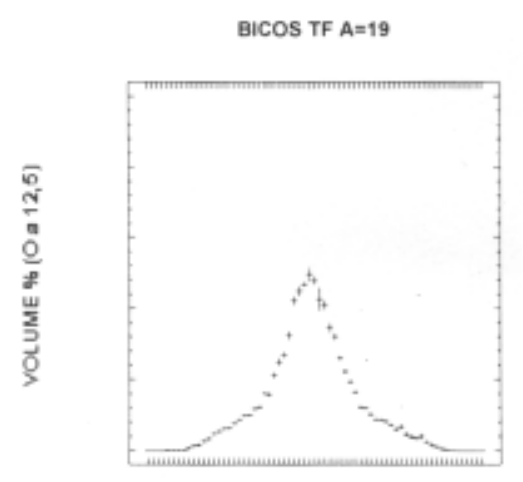

CANALETAS DE 2,5

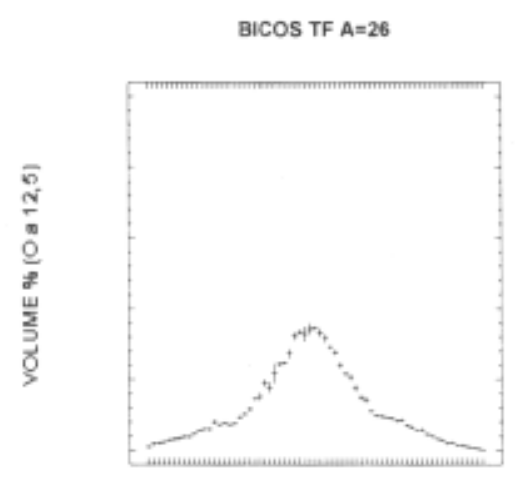

CANALETAS DE 2,5
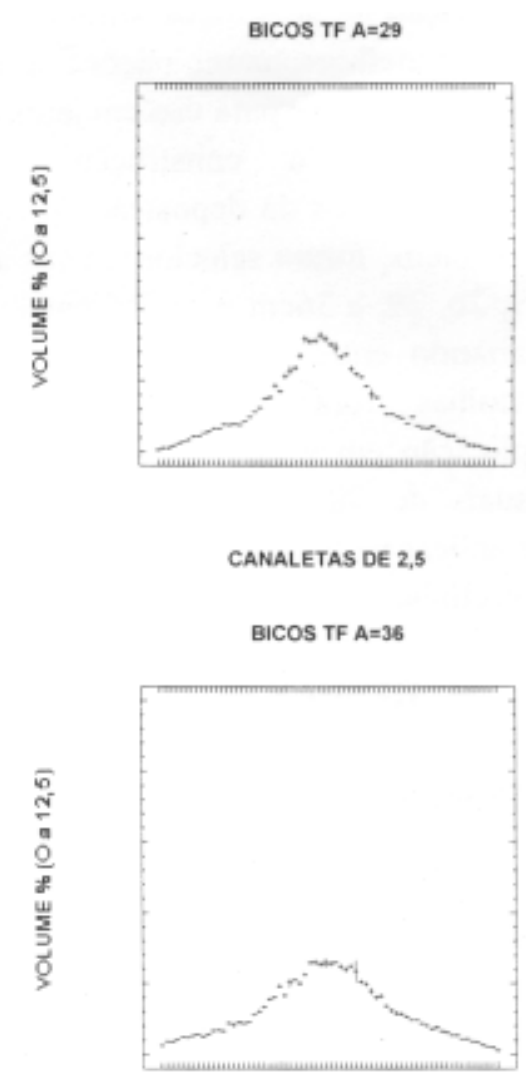

CANALETAS DE 2,5

FIGURA 1. Padrões de distribuição de 10 bicos TF-VS4 em seis alturas $(A=9,16,19,26,29$ e 36cm) com o volume porcentual e respectivo intervalo de $95 \%$ de confiança (se gmento vertical) depositado em cada canaleta de $2,5 \mathrm{~cm}$ (subdivisões da abscissa). A escala da ordenada foi fixada entre 0 e $12,5 \%$, para comparações. 
TABELA 1. Volume percentual médio em canaletas da linha e da entrelinha da cana-de-açúcar, para igualdade da aplicação em volume.

\begin{tabular}{ccc}
\hline \multirow{2}{*}{$\begin{array}{c}\text { Espaçamento da } \\
\text { cana-de-açúcar }(\mathbf{c m})\end{array}$} & \multicolumn{2}{c}{ Volume pulverizado (\%) } \\
\cline { 2 - 3 } 90 & 1,39 & Entrelinha \\
100 & 1,25 & 2,78 \\
110 & 1,14 & 2,50 \\
120 & 1,04 & 2,27 \\
130 & 0,96 & 2,08 \\
140 & 0,89 & 1,92 \\
150 & 0,83 & 1,79 \\
\hline
\end{tabular}

IC $=95 \%, 2$ BICOS TF E $=30$

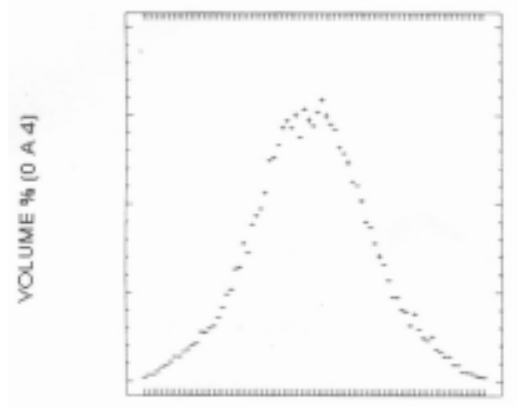

CANALETAS DE 2,5

$I C=95 \%, 2$ BICOS TF E $=45$

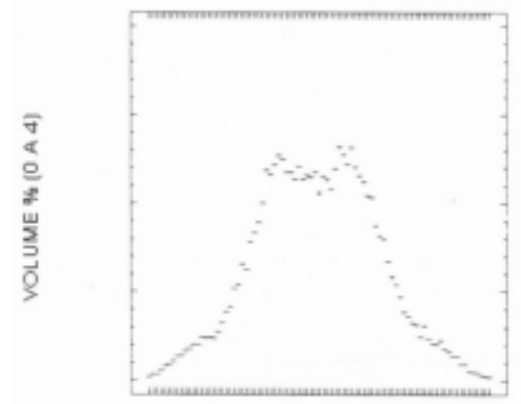

CANALETAS DE 2,5
IC $=95 \%, 2$ BICOS TF $E=35$

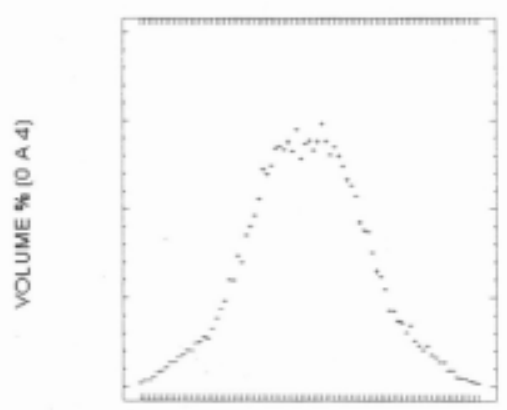

CANALETAS DE 2,5

IC $=95 \%, 2$ BICOS TF E $=50$

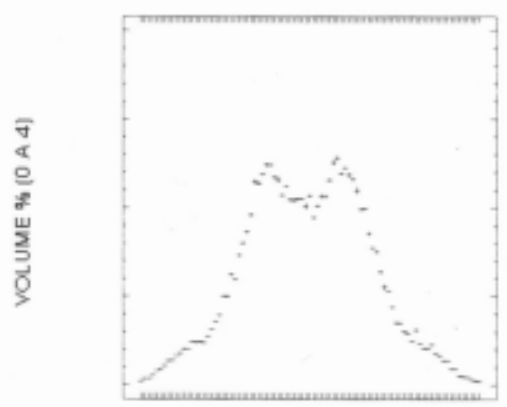

CANALETAS DE 2,5
IC $=95 \%, 2$ BICOS TF $E=40$

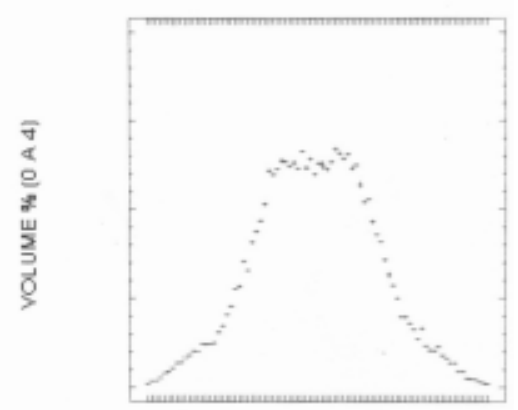

CANALETAS DE 2,5

IC $=95 \%, 2$ BICOS TF $E=60$

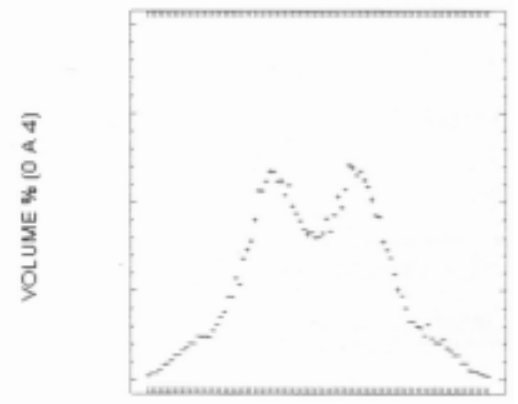

CANALETAS DE 2.5

FIGURA 2. Padrões de distribuição de dois bicos dispostos no centro da entrelinha da cana-de-açúcar, na altura $\mathrm{A}=29 \mathrm{~cm}$ e com espaçamento $(\mathrm{E})$ entre bicos variando de $30 \mathrm{a} 60 \mathrm{~cm}$. 
IC $=95 \%, 2$ BICOS TF E=35

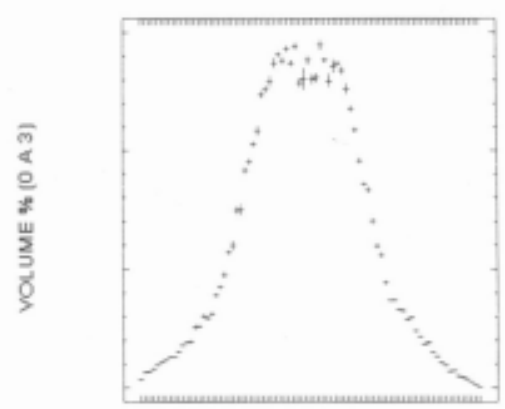

CANALETAS DE 2,5

IC $=95 \%, 2$ Bicos TF $E=45$

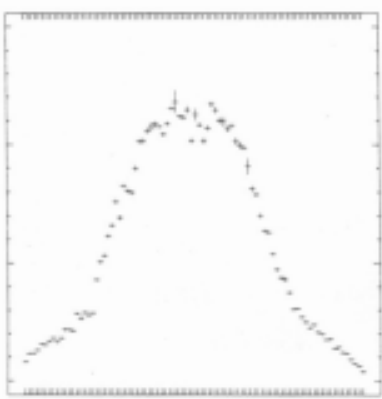

CANALETAS DE 2,5
IC $=95 \%, 2$ BICOS TF E $=40$

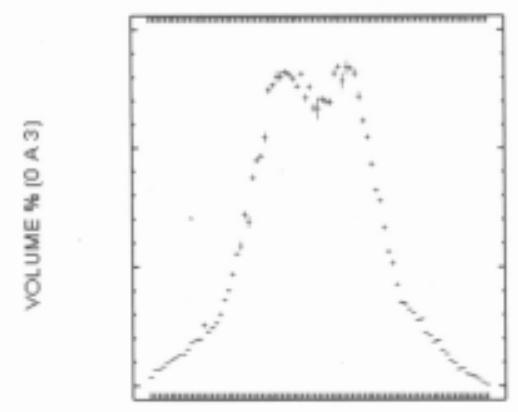

CANALETAS DE 2,5

$I C=95 \%, 2$ Bicos TF E=50

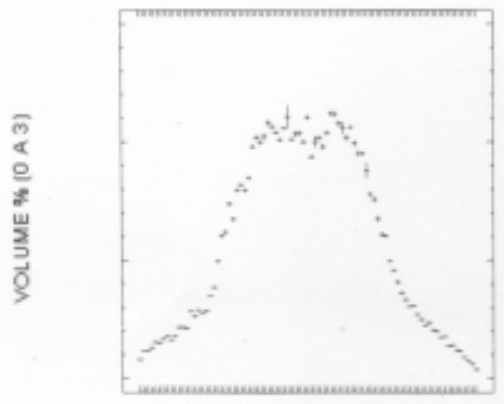

CANALETAS DE 2,5
IC=95\%, 2 BICOS TF E=40

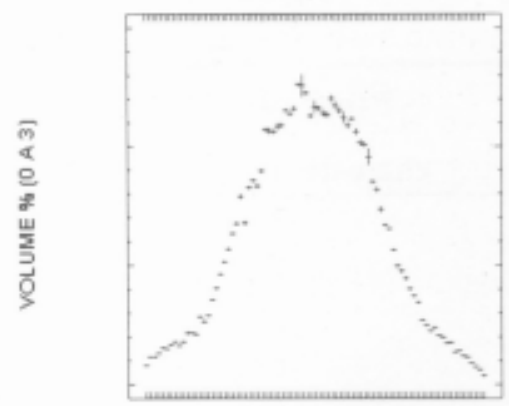

CANALETAS DE 2,5

IC $=95 \%, 2$ BICOS TF E=55

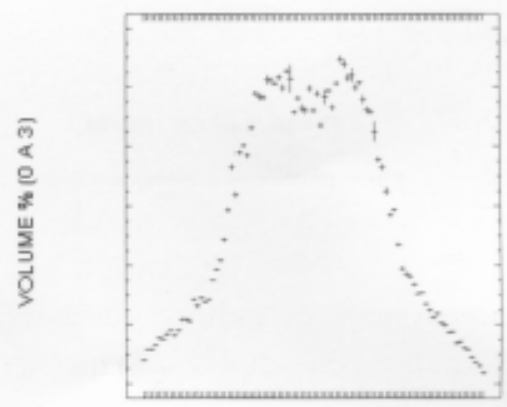

CANALETAS DE 2,5

FIG URA 3. Pad rões de dis tribuiçãa que poderiam ser recomendados para a cana-de-açú car em espaçamentos de 90 a $150 \mathrm{~cm}$, entre as alturas testadas.

TABELA 2. Sugestões de combinações de altura e de espaçamento entre bicos TF-VS4 para uso em canade-açúcar.

\begin{tabular}{cccc}
\hline $\begin{array}{c}\text { Espaçamento da } \\
\text { Cana-de-açúcar (cm) }\end{array}$ & Altura do Alvo (A) & $\begin{array}{c}\text { Espaçamento entre } \\
\text { bicos (E) }\end{array}$ & $\begin{array}{c}\text { Relação } \\
\text { (E/A) }\end{array}$ \\
\hline 90 & 20 & 30 & 1,5 \\
100 & 25 & 35 & 1,4 \\
110 & 30 & 40 & 1,3 \\
120 & 30 & 45 & 1,5 \\
130 & 35 & 45 & 1,3 \\
140 & 35 & 50 & 1,4 \\
150 & 40 & 55 & 1,4 \\
\hline
\end{tabular}


A Figura 2 mostra o efeito do espaçamento entre bicos, qua ndo se fixa uma altura (no caso, $A=29 \mathrm{~cm}$ ). Nota-se a forma mais triangular no espaçamento $\mathrm{E}=30 \mathrm{~cm}$, quando a relação E/A é mais bai xa que a ideal; o suave platô central nos espaçamentos $\mathrm{E}=40$ ou $45 \mathrm{~cm}$, quando a relação E/A se aproxima da ideal, e o aparecimento da "sela" central, quando a relação é maior que a ideal, ver o gráfico de $\mathrm{E}=60 \mathrm{~cm}$, na referida Figura. A análise da Figura 2 sugere que a relação E/A ideal deva situar-se ao redor de 1,4. Em trabalho similar, com bicos TJ60-11006, a relação E/A mostrou-se um pouco menor ao redor de 1,0 (Matuo et al., 1994).

A Figura 3 mostra outras combinações de altura e espaçamento, em complemento às da Figura 2 e que podem ser empregadas para jato dirigido, em cana-de-açúcar.

Com base nos resultados apresentados nas Figuras 2 e 3 podem-se sugerir algumas combinações de altura do alvo (A) e de espaçamento entre bicos (E) que seriam adequadas para se usar na prática com o bico TF-VS4 em cana-de-açúcar (Tabela 2). Portanto, os bicos TFVS4 mostraram vazões média de 2,3 1/min, com coeficiente de variação 2,5\%; os padrões de distribuição ou deposição mostraram uma boa precisão, com um intervalo de confi ança menor que $0,5 \%$ por canaleta, especialmente nas alturas mais baixas; em função dos padrões de distribuição foi possível selecionar as melhores combinações de altura do alvo (A) e espaçamento entre bicos (E), para cada um dos espaçamentos usuais da cana-de-açúcar; a relação E/A ideal se mostrou próxima de 1,4.

\section{LITERATURA CITADA}

LUNSFORD, J. Effect of herbicide rate, additivus, growth stage and competitive crop on the control of Texas pan icum with fluazifop butyl. In: SOUTHERN WEED SCIENCE SOCIETY, Annual Meeting, 36 , USA, 1983. Proceedings, p.149.

MATUO, T. Tecnologia de aplicação de defensivos. In: GRAZIANO NETO, F., ed. Uso de agrotóxico e receituário agronômico. São Paulo, Agroedições, 1982. p.103-106.

MATUO, T., PERESSIN, V.A., PERECIN, D., PIO, L.C., BRAZ, B.A. Aplicação em jato dirigido em cana-de-açúcar. I. Espaçamento en tre bicos Tw in Jet pa ra ap li cação do herbicida MSMA. Eng. agric., v.14, p.6982, 1994.

ORGANIZACIÓN MUNDIAL DE LA SALUD. Material de lucha contra los vectores, Ginebra, OMS, 1976, 189p.

PERECIN, D., PERESSIN, V.A., MATUO, T., BARBOSA, J.C., PIO, L.C., BRAZ, B.A. Padrões de distribuição obtidos com bicos TwinJet, em função da altura e do espaçamento entre bicos. Eng. Agric., v.14, p.19-30, 1994.

SPRAYING SYSTEMS DO BRASIL LTDA. Uso do bico TwinJet em jato dirigido - cana-deaçúc ar. In: Ca na -de-açúc ar: $B i \cos \mathrm{e}$ Acessórios de Barra. São Paulo, Depto Marketing..., Manual BR-CAI, p.20-21, 1995.

SIL VA, J.G. da. Equipamentos e mét odos de aplicação de herbicidas. Inf. Agropec., Belo Horizonte, v.8, n.87, p.44-54, 1982. 